\title{
High precision radial velocity measurements in the infrared ${ }^{\star}$ A first assessment of the RV stability of CRIRES
}

\author{
A. Seifahrt ${ }^{1,2,3}$ and H. U. Käufl ${ }^{3}$ \\ 1 Universität Göttingen, Institut für Astrophysik, Friedrich-Hund-Platz 1, 37077 Göttingen, Germany \\ e-mail: seifahrt@astro.physik.uni-goettingen.de \\ 2 Universität Jena, Astrophysikalisches Institut und Universitäts-Sternwarte, Schillergässchen 2, 07745 Jena, Germany \\ 3 ESO, Karl-Schwarzschild-Str. 2, 85748 Garching, Germany
}

Received 10 May 2008 / Accepted 5 September 2008

ABSTRACT

\begin{abstract}
High precision radial velocity (RV) measurements in the near infrared are in high demand, especially in the context of exoplanet search campaigns shifting their interest to late type stars in order to detect planets with ever lower mass or targeting embedded pre-main-sequence objects.

ESO commissioned a new spectrograph at the VLT - CRIRES - designed for high resolution near-infrared spectroscopy with a comparably broad wavelength coverage and the possibility of using gascells to provide a stable RV zero-point.

Here, we investigate the intrinsic short-term RV stability of CRIRES, both with gascell calibration data and on-sky measurements using the absorption lines of the Earth's atmosphere imprinted in the source spectrum as a local RV rest frame. Moreover, we also investigate for the first time the intrinsic stability of telluric lines at $4100 \mathrm{~nm}$ for features originating in the lower troposphere.

Our analysis of nearly $5 \mathrm{~h}$ of consecutive observations of MS Vel, a M2II bright giant centred on two SiO first overtone band-heads at $4100 \mathrm{~nm}$, demonstrates that the intrinsic short-term stability of CRIRES is very high, showing only a slow and fully compensateable drift of up to $60 \mathrm{~m} / \mathrm{s}$ after $4.5 \mathrm{~h}$. The radial velocity of the telluric lines is constant down to a level of approx. $\pm 10 \mathrm{~m} / \mathrm{s}$ (or 7/1000 of one pixel). Utilising the same telluric lines as a rest frame for our radial velocity measurements of the science target, we obtain a constant RV with a precision of approx. $\pm 20 \mathrm{~m} / \mathrm{s}$ for MS Vel, as expected for a M-giant.
\end{abstract}

Key words. instrumentation: spectrographs - instrumentation: adaptive optics - methods: observational techniques: radial velocities - techniques: spectroscopic - stars: AGB and post-AGB

\section{Introduction}

CRIRES $^{1}$ is a newly commissioned adaptive optics (AO) fed spectrograph at UT1 (Antu) of the Very Large Telescope on Paranal, Chile. The spectrograph provides a resolution of up to 100000 at $960-5200 \mathrm{~nm}$ with an instantaneous wavelength coverage of $\sim \lambda / 50$ on a $4096 \times 512$ pixel detector mosaic (Käufl et al. 2004, 2006b). A MACAO2 adaptive optics system (Arsenault et al. 2004) provides diffraction limited images at the entrance slit of CRIRES with Strehl ratios of up to $65 \%$ in the $K$ band, thus roughly doubling the throughput under reasonable seeing conditions for the nominal $0.2^{\prime \prime}$ slit when compared to seeing-limited performance (Paufique et al. 2006).

The spectrum, being a full order at the shortest wavelength in the $J$ band and about $1 / 5$ of an order at the longest wavelength in $M$ band, is imaged in long slit mode onto a mosaic of four Aladdin III detectors with a gap of $\sim 250$ pix between the chips. Thus, the instantaneous wavelength coverage is much shorter than for optical cross-dispersed spectrographs. However, the gain in efficiency for RV measurements, primarily dependent on spectral coverage and $\mathrm{S} / \mathrm{N}$, will shift in favour of CRIRES when the effective SED of the science target peaks longwards of $900 \mathrm{~nm}$ where other optical spectrographs commonly used for

\footnotetext{
* Based on observations at the European Southern Observatory, Paranal, during commissioning of CRIRES.

${ }^{1}$ CRIRES stands for $\underline{C}$ yogenic InfraRed Echelle Spectrograph.

${ }^{2}$ MACAO stands for Multiple Application $\underline{\text { Curvature }}$ Adaptive Optics.
}

RV measurements do either not operate (e.g. HARPS) or dramatically drop in sensitivity (e.g. UVES).

For precise RV measurements the spectrograph has to be well characterised since we deal with various sources of uncertainties that could induce wavelength shifts in the measured spectra. Since CRIRES is a cryogenic, thus actively stabilised spectrograph, temperature drifts are small but certainly not negligible. The same is true for vibrations and flexure, especially due to changing thermal gradients in the structure. Being mounted on a Nasmyth platform of the VLT, the system is intrinsically very stable but vibrations induced by the cryogenic cooling system can be suspected to cause instability.

A second effect is the imperfect repeatability of dispersive parts on moving functions subject to slip-stick effects: the prism (pre-disperser), the intermediate slit and the grating. It is forseen that these functions are left untouched when the same instrumental setup is used in subsequent observations. However, for observations that span days to months, the repeatability of the setup could be an issue, if it cannot be well calibrated.

Also, a long-slit spectrograph suffers the natural problem of alignment uncertainties of the source with respect to the slit centre. For spectrographs fed by an adaptive optics system the situation is worse in some aspects:

1. The FWHM of the source is usually smaller than the slit width;

2. The alignment of the adaptive optics with respect to the entrance slit of the spectrograph suffers instabilities, requiring 
an active slit-viewer guiding in order to keep the source centred on the slit;

3. The algorithm for slit-viewer guiding can only work on the wings of the stellar PSF, since its core is masked by the slit;

4. The refraction caused by the Earth's atmosphere has to be measured and compensated for simultaneously with observations at three different wavelengths: in the optical for the AO wave front sensor, in $J, H$ or $K$ band for the slit-viewer (used for active guiding) and at the respective wavelength of the observation, in our case at $4100 \mathrm{~nm}$.

All these effects will cause a degraded stability and a possible drift of the wavelength scale with time. We thus aimed to characterise the system and the means to calibrate it and test the on-sky performance in terms of RV stability during a single night.

For CRIRES a RV precision of $15 \mathrm{~m} / \mathrm{s}$ would require pointing and tracking with a precision of one milli arcsec (mas), given that one pixel in the dispersion direction $\left(1.5 \mathrm{~km} \mathrm{~s}^{-1}\right)$ equals 100 mas at the entrance slit of the spectrograph. The tracking precision is limited by the amount of light in the wings of the PSF on which the guiding algorithm of the slitviewer operates. Tracking the core of the PSF with the seeing-limited wings is a general limitation. Even for bright sources, the performance of the guiding algorithm is not sufficient to stabilise the source in the slit with a precision better than approx. 25 mas (approx. $200 \mathrm{~m} / \mathrm{s}$ ), which makes a reference source (telluric or gascell absorption lines) indispensable when aiming for high precision RV measurements.

\section{Observations}

For our on-sky test we were looking for a bright target which should present no significant RV signal within a few hours. We chose the M2II star MS Vel (Egret 1980), a rather bright $\left(K \mathrm{~s}=3.7 \mathrm{mag}\right.$ (DENIS), $F_{4.29 \mu \mathrm{m}}=16 \mathrm{Jy}$, Egan et al. 2003) semi-regular variable star (Adelman 2001) with a proposed period of about 360d (Makarov et al. 1994). We have re-analysed HIPPARCOS and TYCHO photometry simultaneously covering about 3 years and about 5.5 years worth of data from the ASAS3 $V$ band survey (Pojmanski 2002) using a Lomb-Scargle Periodogram to identify variability in MS Vel. All three data sets show a strong period of $P=360 \pm 5 \mathrm{~d}$ with a somewhat larger value $(P \sim 363.5 \mathrm{~d})$ for the HIPPARCOS and TYCHO epochs of 1990-1993 and a shorter value $(P \sim 355 \mathrm{~d})$ in the full epoch covered by the ASAS data (2000-2006). This dominant period with an amplitude of about $\Delta V \simeq 0.8$ mag is accompanied by shorter periods of 18,26 and 28 days with much smaller amplitudes in the earlier data which disappear however in the much better sampled data from the later epoch. Conversely, periods of 155 and 185 days, present in the ASAS data cannot be found in the HIPPARCOS and TYCHO data. All these periods have false-alarm-probabilities of much less than $0.1 \%$ and are therefore real, even though not stable. We see that MS Vel is variable on various time-scales of weeks to a about a year. However, it is very unlikely that the object shows a significant RV signal in only a few hours, given its large radius. As predicted by 3D simulations (Freytag \& Höfner 2008), typical photometric periods for early to mid M-type giants are of the order of several dozen to a few hundred days (Percy et al. 2008). Radial velocity surveys of K-type giants also revealed periods on the same timescale (Hekker et al. 2008). Short-term oscillations on M-type giants are rarely observed and still show periods in excess of one day (Koen \& Laney 2000).
We have observed MS Vel on the night of 2007, Feb. 02 from 03:23 to 07:56 UT. Observations were obtained with the adaptive optics loop closed and locked on the science target. Strehl ratios of more than $50 \%$ could be achieved for the majority of the program, delivering most of the encircled energy in the diffraction limited core with a FWHM of approx. 100 mas. The DIMM seeing was $1^{\prime \prime}$ on average, with very good conditions $\left(0.65^{\prime \prime}-1.0^{\prime \prime}\right)$ for the first three hours and slightly worse conditions $\left(1.0^{\prime \prime}-1.5^{\prime \prime}\right)$ for the last $90 \mathrm{~min}$. A slit width of $0.4^{\prime \prime}$ was chosen, providing a nominal resolution of $R \simeq 50000$. The total flux in the spectrum shows a weak dependence on the seeing, with a degradation of about $20-30 \%$ in flux at the end of the campaign, as expected when the atmospheric conditions worsen and the Strehl ratio drops, closely matching the predictions of the exposure time calculator (ETC) for our case.

We chose a setting at $\lambda_{\text {Ref }}=4029.9 \mathrm{~nm}$ to cover two $\mathrm{SiO}$ band-heads at $4043 \mathrm{~nm}\left({ }^{28} \mathrm{Si}^{16} \mathrm{O}, v=3 \rightarrow 1\right)$ and $4084 \mathrm{~nm}$ $\left({ }^{28} \mathrm{Si}^{16} \mathrm{O}, v=4 \rightarrow 2\right)$. The range of the $\mathrm{SiO}$-overtone at $4000-4100 \mathrm{~nm}$ is a well understood but still rich line system (Aringer et al. 1999) in a region where photospheres are otherwise generally clean while the Earth's atmosphere provides a rich line system of $\mathrm{N}_{2} \mathrm{O}$, originating mainly from the lower troposphere.

An AAA-BBB-BBB-AAA nodding pattern with individual integration times of $10 \mathrm{~s}$ per frame was used. The nodding amplitude was set to $10^{\prime \prime}$. The average $\mathrm{S} / \mathrm{N}$ in each single spectrum was found to be approx. 200.

After each cycle the instrument switched to calibration mode to take a single, short spectrum of the $\mathrm{N}_{2} \mathrm{O}$ gascell $(10 \mathrm{hPa}$, $140 \mathrm{~mm}$ length), illuminated by a blackbody involving an Ulbricht sphere. The slit width was held constant for the science and calibration measurements. Since the light from the gascell is a spatially flat source, the entrance slit of CRIRES is evenly illuminated and the $0.4^{\prime \prime}$ slit delivers a resolution of approx. 50000 . No change in illumination is expected throughout the length of the program, making these calibration measurements a suitable reference for the intrinsic stability of the spectrograph, independent of slit centring issues of the actual science target. After completing such a set of observations we re-acquired the object in the slit-viewer, closed the AO loop again and started a new cycle of on-sky observations. This procedure was repeated 25 times.

During this series of observations, the dispersive elements of CRIRES - the prism (pre-disperser), intermediate slit and the grating - where electronically de-mobilised and held in fixed positions. This precaution was taken to avoid accidental repositioning of functions. Thus, the observations of the calibration source were only affected by temperature drifts and flexure. Besides this non standard setup the observations made use of ESO observatory standard procedures.

Both the signal-to-noise ratio and the spectral resolution are of critical importance for RV studies. While both quantities are closely connected to the slit width of regular long-slit spectrographs, the situation for CRIRES is more complex. Since CRIRES works with an underfilled entrance slit, the achievable resolution is not strictly depending on the chosen slit width but is mainly governed by the FWHM of the stellar PSF in the slit, hence also depends on the AO performance and the wavelength of the observation. The troughput is only marginally dependant on the slit width in the regime of potentially high Strehl ratios in $K, L$, and $M$ band, reaching over $80 \%$ at the longest wavelength under favourable conditions (see the CRIRES ETC and Paufique et al. 2006). The typical diffraction limited FWHM of the VLT at $4000 \mathrm{~nm}\left(\sim 0.1^{\prime \prime}\right)$ is much smaller than the minimum 

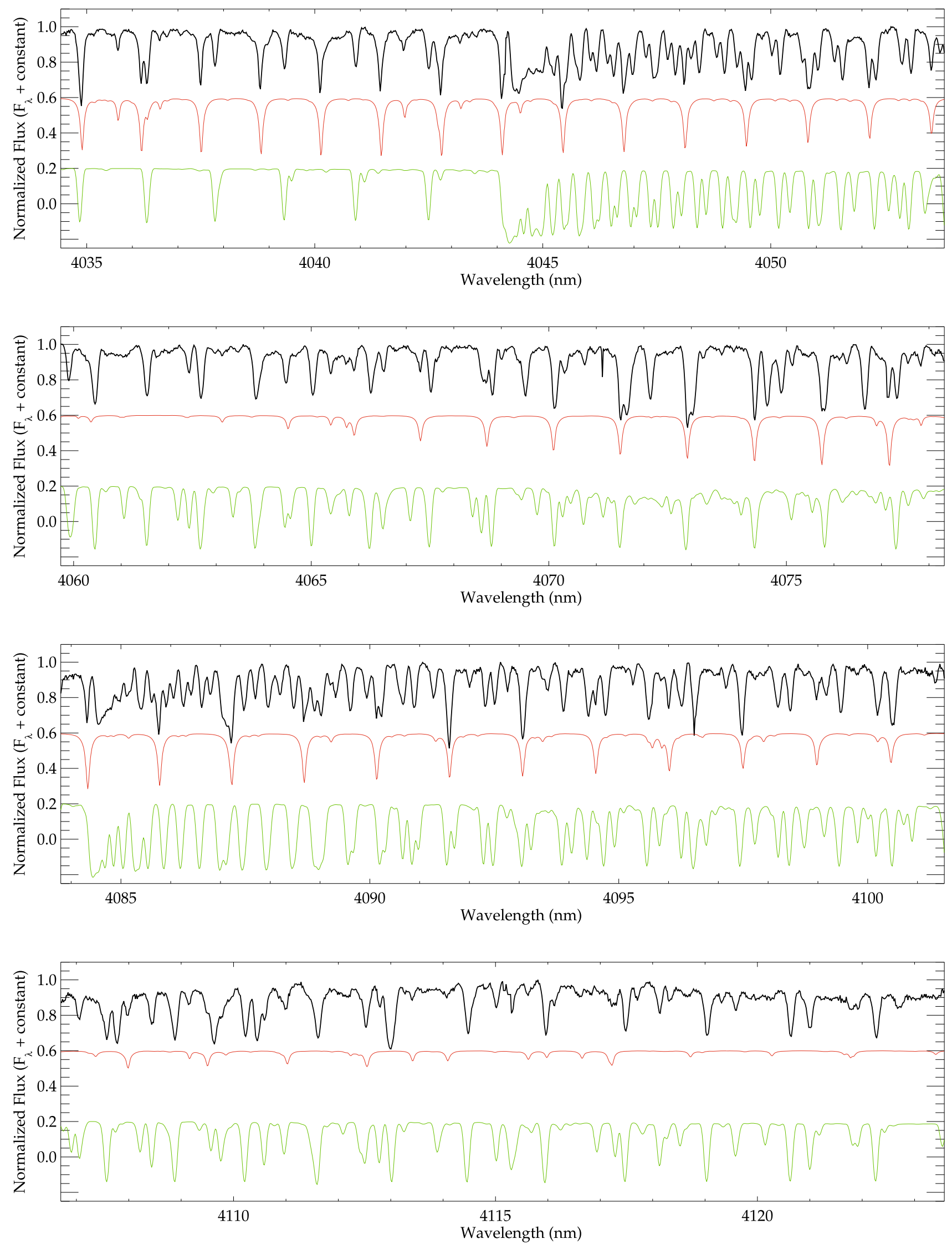

Fig. 1. Spectrum of MS Vel and the Earth's atmosphere on the four chips of CRIRES, displayed in four viewgraphs. Shown in each viewgraph: measured spectrum (top, black line), telluric model (center, red line, shifted by -0.4 ) and PHOENIX model spectrum for MS Vel: $T_{\text {eff }}=3500 \mathrm{~K}$ and $\log g=0.5$ (bottom, green line, shifted by -0.8 ). See text for details. 

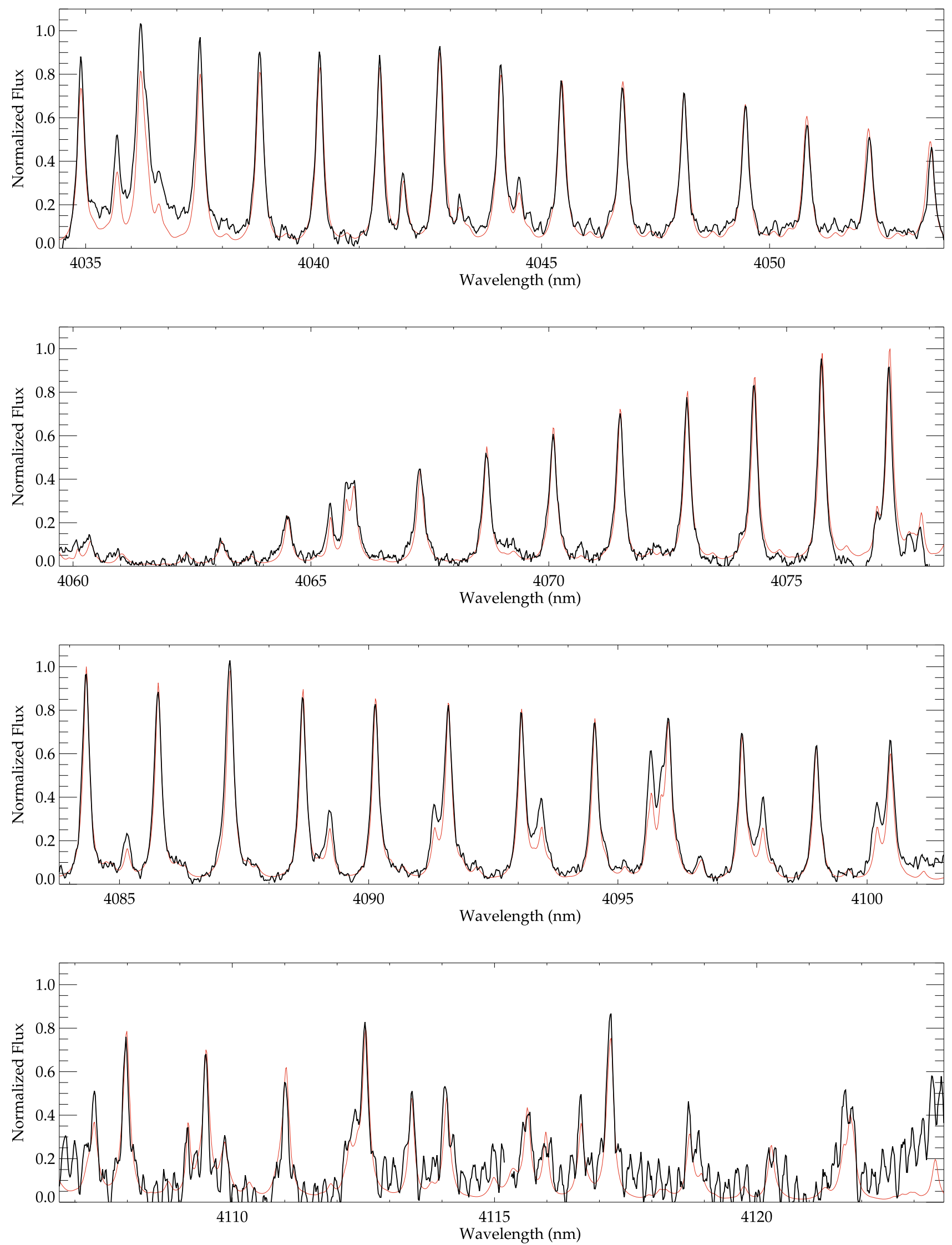

Fig. 2. Spectrum of the Earth's atmosphere in emission (sky background) on the four chips of CRIRES, displayed in four viewgraphs. The relative strength of the emission bands follows the behavior of the $\mathrm{N}_{2} \mathrm{O}$ absorption bands shown in Fig. 3. The relative intensities on chips 2,3 , and 4 are scaled up by factors of $7 \%, 15 \%$ and $150 \%$, respectively, relative to chip 1 . Over-plotted is a synthetic emission spectrum (red line). Note that the isolated $\mathrm{H}_{2} \mathrm{O}$ lines appear much stronger in emission than they appear in absoption in Fig. 1. See text for further details. 

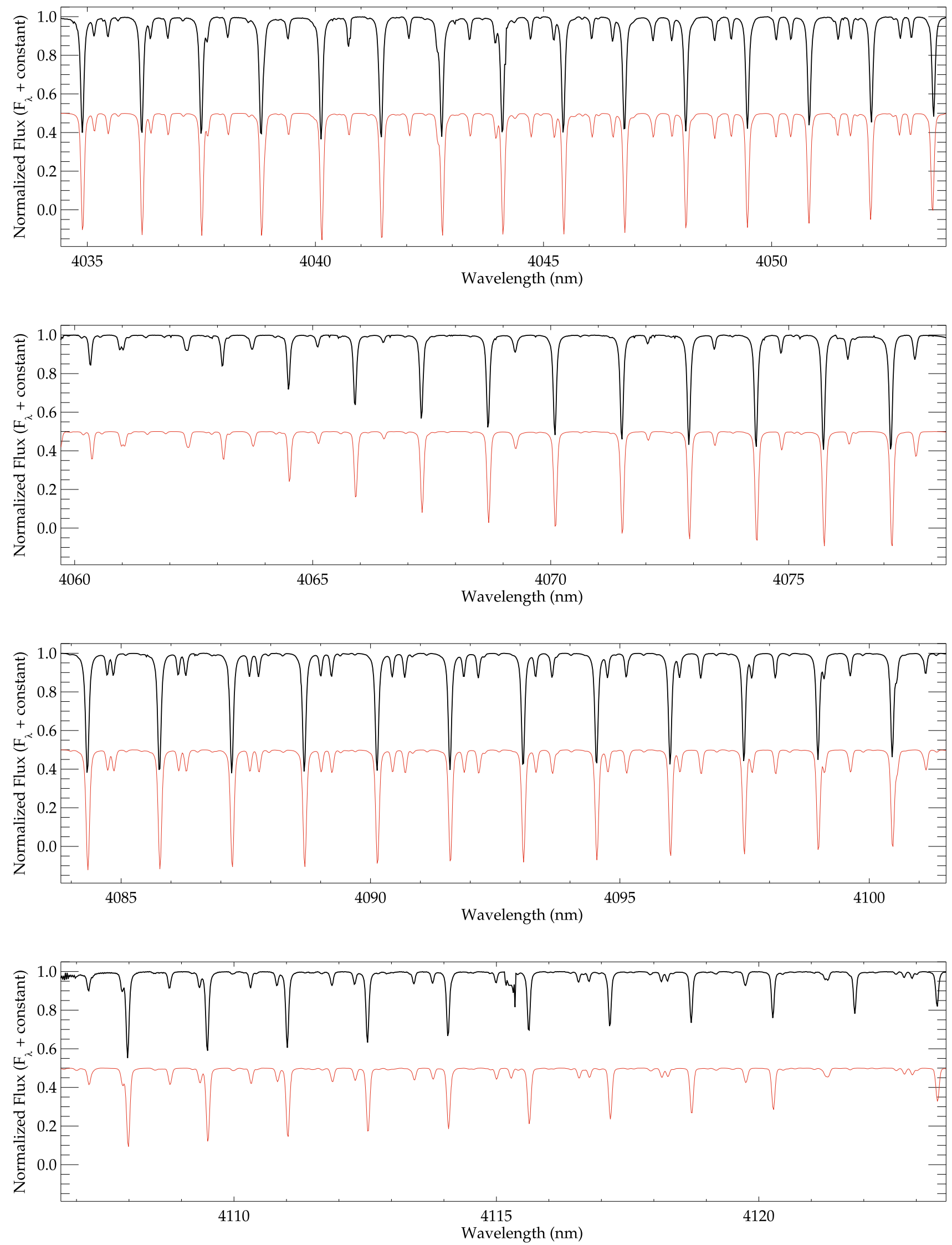

Fig. 3. Spectrum of the $\mathrm{N}_{2} \mathrm{O}$ gascell, illuminated by the flat-field lamp in calibration mode. Overplotted is a synthetic spectrum (red line, offset -0.6), calculated with FASCODE and HITRAN. See text for details. 
slit width and the spectral resolution is limited by the optics of the spectrograph and the stability of the source in the slit during an integration rather than by the width of the profile alone or the width of the entrance slit. The true resolution achieved with the $0.4^{\prime \prime}$ slit is thus likely closer to 100000 than to its lower limit of 50000 , defined by the slit width. Closing the slit to $0.2^{\prime \prime}$ would only marginally improve the resolution by basically masking some light from the seeing limited halo of the PSF. Likewise, the throughput of the $0.4^{\prime \prime}$ slit is only about $20 \%$ higher than for the $0.2^{\prime \prime}$ slit in our case.

However, choosing a wide slit further decreases the amount of reflected light available for the guiding algorithm of the slitviewer and further increases the chance of misplacements and instabilities of the source position in the slit. Since it is expected that future RV programs conducted with CRIRES will also observe rather faint targets under low Strehl ratios, a regime where a large slit width will more notably improve the efficiency, we nevertheless decided to use the $0.4^{\prime \prime}$ slit to investigate these effects in this first study.

\section{Data reduction}

\subsection{Basic treatment and spectral extraction}

For data reduction the raw data were divided by a flat-field frame, mainly to correct for a fixed pattern effect of the detector array and to normalise the continuum to a satisfactory level. To subtract the background emission we subtracted the mean of the three frames from the next nodding position from the current frame, leaving a flat sky background around the source spectrum. The spectra were than extracted using the optimum extraction algorithm of the CRIRES data reduction pipeline.

Spatially extended sources, such as the sky background as well as the spectra of the gascell in the respective calibration frames were extracted after flat-fielding of the raw frames and integrating over a spatial range of 100 pixels between the two nodding positions of the source. We thus sample the same region that is covered by the source spectrum itself, without introducing offsets caused by slit curvature, detector residual tilt, and distortion, as we will discuss in Sect. 4.

After this treatment we have 300 individual spectra of the object and of the sky background emission at our disposal. In addition we have 25 spectra, one for each cycle of observations, of the $\mathrm{N}_{2} \mathrm{O}$ gascell. Hence, we have four systems of spectral lines that we can analyse here:

1. The photospheric lines of the target, MS Vel, foremost being the two $\mathrm{SiO}$ band-heads and various unidentified lines,

2. The telluric lines in absorption, imprinted on the spectra of the target,

3. The telluric lines in emission as a spatially extended background source along the whole slit (note: due to Kirchhoff's law the atmospheric absorption lines become inverted into emission lines when viewed against the night sky),

4. The $\mathrm{N}_{2} \mathrm{O}$ lines from the gascell, also as a spatially extended source since the instrument's flat-field source was used to illuminate the gascell.

All four line systems can now be analysed by cross-correlation to measure the zero-point shift of the wavelength in each of the spectra.

\subsection{Methodology}

In the case of the spectrum of the star being taken through a gascell, the classical approach to measure radial velocities is to cross-correlate the product of two template spectra (normally the stellar spectrum and the spectrum of the gascell) with the actual measurement. This requires template spectra of high quality. Unfortunately no template spectra for a M2II giant at $4100 \mathrm{~nm}$ of sufficient quality was available for us. A test with a synthetic spectrum from the library of PHOENIX (Hauschildt \& Baron 2005, and references therein) spectra $^{3}$, as displayed in Fig. 1, did not lead to consistent results when used as a template for the cross-correlation analysis, since the line lists used to compute the synthetic spectra are not precise enough and show notable shifts of the different species present in late type giants at $4100 \mathrm{~nm}$.

For the telluric lines, however, we have access to quite precise synthetic spectra of the Earth's atmosphere. Using a standard atmospheric model for the Paranal site and adopting a typical humidity for the time of the observation we can construct a synthetic spectrum at any given wavelength using the FASCODE algorithm (Clough et al. 1981, 1992), a line-byline radiative transfer model for the Earth's atmosphere, and HITRAN (Rothman et al. 2005) as a database for molecular transitions. A spectrum of the Earth's atmosphere in our wavelength window is shown in Fig. 1 for the telluric lines in absorption as seen against the stellar continuum. In Fig. 2 we show the same lines in emission (line radiation against dark sky background). The predominant species in the observed spectral range is $\mathrm{N}_{2} \mathrm{O}$, the same species as in our gascell. $\mathrm{N}_{2} \mathrm{O}$ shows nearly regular spaced lines of the $P$-branch on chips two, three, and four and of the $R$-branch on chip one. Additional minor contributions arise from some isolated water vapour lines. These lines form in lower, thus warmer parts of the atmosphere and are stronger in emission than in absorption compared to the neighboring $\mathrm{N}_{2} \mathrm{O}$ lines. They are thus more prominent in the sky emission spectrum (Fig. 2) than in its "inverse" counterpart, the telluric absorption spectrum (Fig. 1).

A more detailed discussion of the properties of the telluric lines and how to use them as a wavelength standard will be given in a later paper.

Similarly to the telluric features, we can compute the spectrum of our gascell, a $\mathrm{N}_{2} \mathrm{O}$ spectrum at room temperature and $\sim 10 \mathrm{hPa}$ pressure. The result is shown in Fig. 3 . These transitions are calibrated in frequency with heterodyne techniques against the time standard (Maki \& Wells 1992), so that they provide an absolute precision, traceable to the time standard, not yet available for optical spectroscopy ( $\mathrm{Li}$ et al. 2008).

\subsection{Wavelength calibration}

For a RV measurement we need to determine the wavelength of each pixel with high precision. The gascell delivers a $\mathrm{N}_{2} \mathrm{O}$ absorption spectrum of high $\mathrm{S} / \mathrm{N}$ with nearly evenly spaced lines distributed in high density on all four chips (see Fig. 3). Using the aforementioned synthetic spectrum of our gascell, we fitted a quadratic solution independently to each of the four chips to match the measured spectrum with the synthetic spectrum, thus providing the zero point and dispersion for the four chips in the first recorded $\mathrm{N}_{2} \mathrm{O}$ spectrum during our measurements.

\footnotetext{
${ }^{3}$ Kindly provided by Peter Hauschildt, Hamburg.
} 


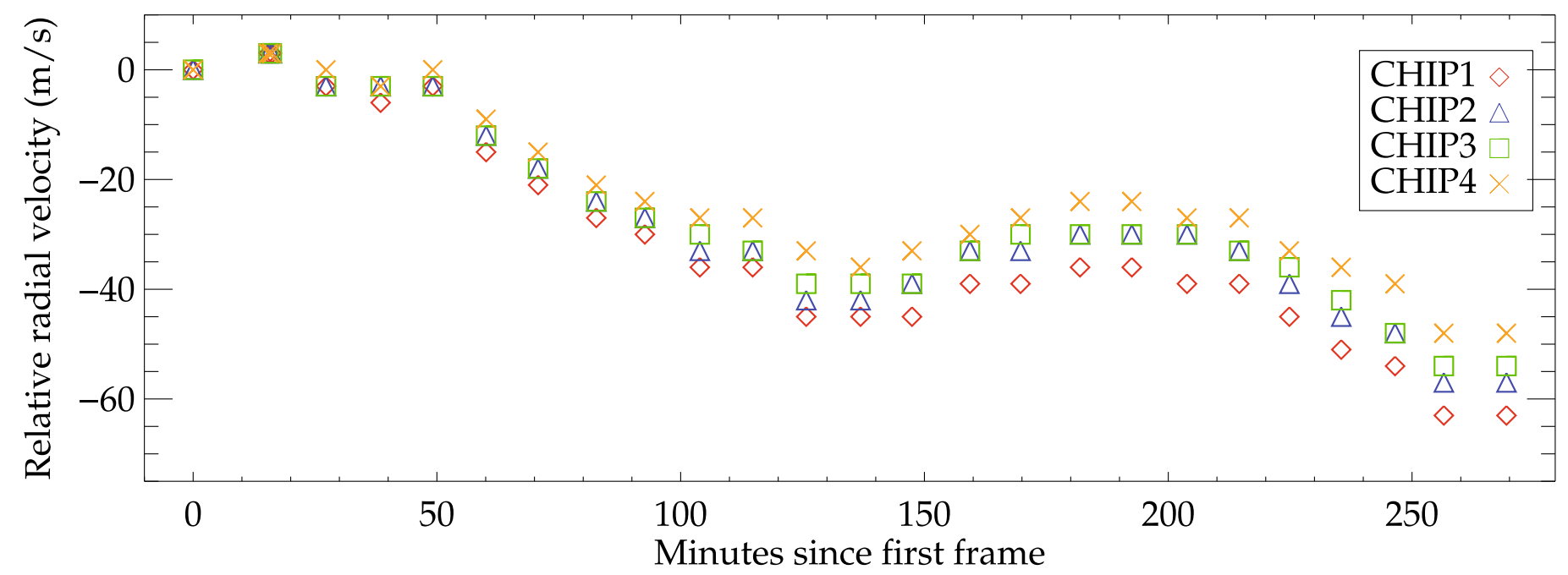

Fig. 4. Apparent radial velocity of the $\mathrm{N}_{2} \mathrm{O}$ gascell line system (see Fig. 3; displayed separately for each chip) observed in calibaration mode (the gascell being illuminated by the flat field lamp). Note the change in dispersion with time which manifests as divergent trends in the RV measured on the four chips. See text for details.

\subsection{Cross-correlation}

Given the lack of a proper template spectrum for the science target, we use the following approach to determine the radial velocity of each of the line systems as outlined above: (1) For the photospheric lines of MS Vel we mask the telluric lines against transmission below $95 \%$ and cross-correlate each of the 300 spectra against the first one obtained. (2) The wavelength shifts of all other line systems - the telluric absorption lines in the spectrum of MS Vel, the sky-background emission lines and the $\mathrm{N}_{2} \mathrm{O}$ absorption lines - are computed by cross-correlating our measured spectra against the respective synthetic model spectrum.

We also tested the methodology used for the photospheric lines of MS Vel (cross-correlation of all spectra against the first one) for the $\mathrm{N}_{2} \mathrm{O}$ lines and the telluric lines. We obtained the same results as for the cross-correlation with the synthetic templates, but find an enhanced scatter due to the lower signal-to-noise ratio when cross-correlating to a measured spectrum instead of against a noise-free model.

\section{Results and discussion}

The 25 calibration spectra of the $\mathrm{N}_{2} \mathrm{O}$ gascell are distributed in time over the full length of our experiment. These spectra probe the intrinsic stability of the spectrograph, since the illumination of the gascell (and thus of the entrance slit) is spatially flat and we do not trace any slit alignment effects. Moreover, the temperature of the gascell, even though not actively stabilised, is certainly not variable to a level that could induce line broadening and line-shifts in the $\mathrm{N}_{2} \mathrm{O}$ spectrum ${ }^{4}$. Hence, we adopt this spectrum as a true rest frame. As can be seen in Fig. 4, the spectral lines of the gascell show a slow drift in wavelength that culminate in a RV offset of $\sim 60 \mathrm{~m} / \mathrm{s}$ after $4.5 \mathrm{~h}$. The drift is

\footnotetext{
${ }^{4}$ During our observations the external temperature as logged by the observatory dropped from 14.8 to $13.3 \mathrm{C}$. The gascell has some thermal inertia, but will ultimately follow the external temperature. Assuming a typical pressure induced frequency shift of $100-150 \mathrm{MHz}$ for $1000 \mathrm{hPa}$ (Deming et al. 1987), even the maximum temperature change for Paranal $\left(-5 \leq T_{\mathrm{amb}} \leq 20 \mathrm{C}\right)$ will not result in a shift of more than $150 \mathrm{kHz}$ of the line frequencies, equivalent to a velocity error of $50 \mathrm{~cm} / \mathrm{s}$.
}

smooth but non-monotonous and is very similar for all four chips leading only to a minimal degree of dispersion change, as can be seen by the slightly diverging RV trends of the four chips. Such a change in dispersion can be assigned to a small drift in the temperature of the grating. At the time of our measurements, the grating was only passively stabilised and the temperature sensor at the grating had a limited resolution of only $\sim 0.1 \mathrm{~K}$. Moreover, the sensor was not part of an active temperature stabilisation of the grating. Following the basic grating equation for echelle spectrographs, the angular dispersion changes linearly with the groove spacing of the grating (induced e.g. by thermal expansion of the grating material) when the setup is otherwise held constant. Adopting a typical expansion coefficient of $\mathrm{d} l / l \simeq 4 \times 10^{-6} \mathrm{~K}^{-1}$ for the grating material, a temperature drift of only $50 \mathrm{mK}$ over $4.5 \mathrm{~h}$ could have caused the observed drift of $60 \mathrm{~m} / \mathrm{s}$. Such a drift would have easily passed the sensor unnoticed. This shortcoming has been fixed. The grating is now actively stabilised to $1 \mathrm{mK}$. The expected wavelength stability is thus approx. $1 \mathrm{~m} / \mathrm{s}$.

This drift would be seen in any spectral line that is intrinsically stable and used as a local rest frame and can thus be compensated for. Ideally the gascell should be used simultaneously while measuring the spectra of the science target, the absorption lines of the gas being imprinted into the source spectrum. However, the chosen gas has to match the spectrum of the object and the gas should not be present in the Earth's atmosphere to avoid any overlap or mixing of the spectrum of the Earth's atmosphere and the spectrum of the gascell. At the time of our experiment we had only one gascell ready and its content, $\mathrm{N}_{2} \mathrm{O}$, is also highly abundant in our atmosphere. Hence, we decided to use the gascell only in calibration mode, illuminated by the flatfield source, to trace the internal stability of the spectrograph and use the atmospheric lines, seen in absorption in the target spectrum, as our local rest frame.

Conversely, we can use the atmospheric lines in emission to trace the behaviour of these lines - to check for their stability. Here the same argument holds as for the $\mathrm{N}_{2} \mathrm{O}$ lines: the atmospheric lines in emission are a spatially flat source unaffected by any slit misalignments. The frequency of the atmospheric lines can be systematically shifted if a net radial velocity is introduced by wind or turbulence. Moreover, these lines will be affected 


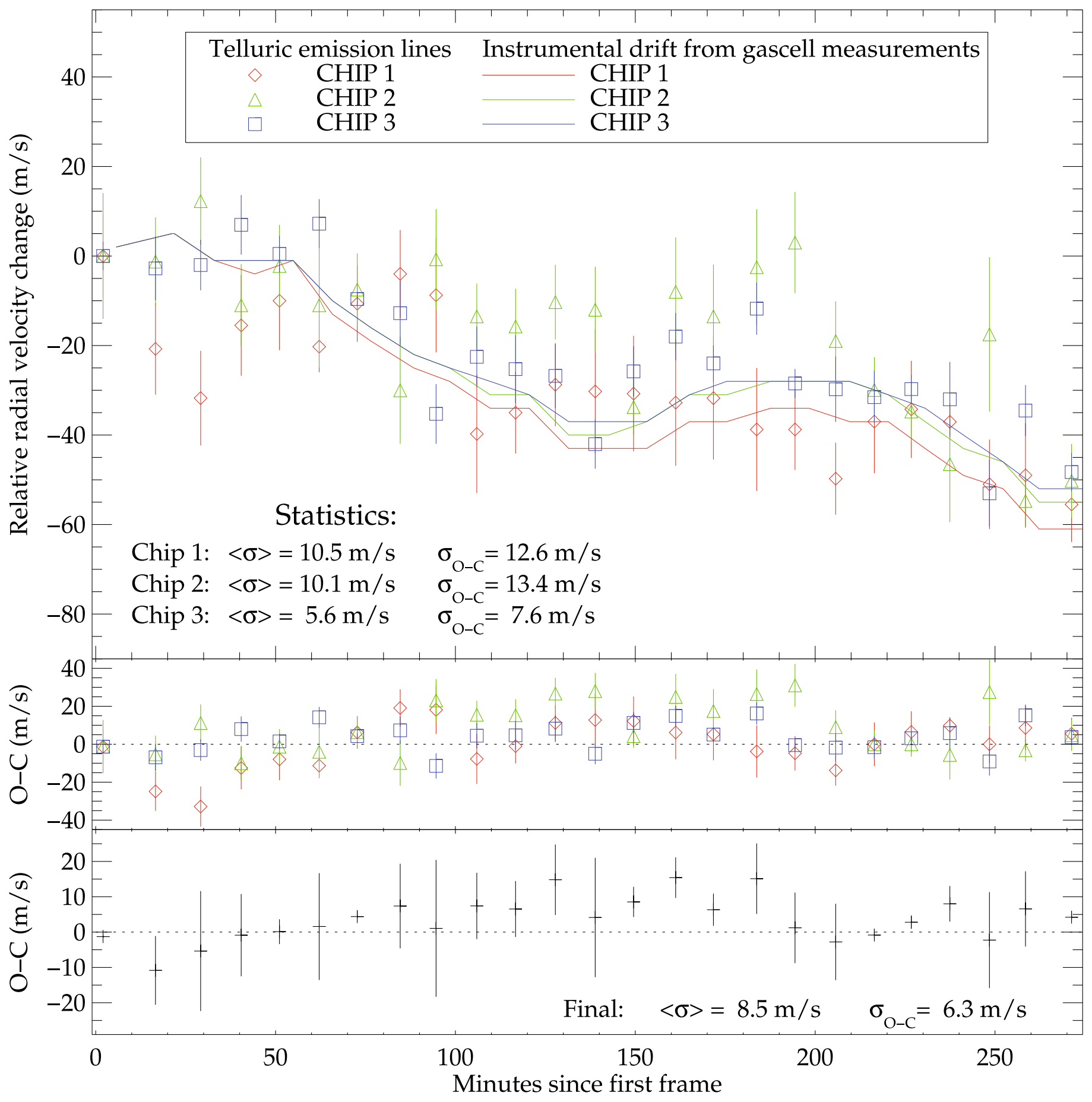

Fig. 5. Radial velocity of the telluric emission lines (see Fig. 2) and the $\mathrm{N}_{2} \mathrm{O}$ gascell line system (see Fig. 3) showing the instrumental drift during the measurement. In the subpanels the difference between both measurements is shown in terms of observed - computed (O-C), first separately for each of the three chips analyzed here, then for the mean of all three chips. The standard deviation of the mean was used to derive formal error bars. Residuals are the final uncertainty for the RV stability of the telluric lines. See text for details.

by the slow drift of the spectrograph itself, measured with the $\mathrm{N}_{2} \mathrm{O}$ gascell lines. In Fig. 5 we show the RV trend of the atmospheric lines in emission. The measured RVs from 12 spectra taken within one nodding cycle are averaged to a single data point. The standard deviation of the mean is shown as the formal $1 \sigma$ error of each data point. We over-plot the drift of the spectrograph and subtract this drift from the RV points. Combining the results from all three chips where atmospheric lines are present results in a flat RV distribution over time with residuals (rms) of the order of $\leq 10 \mathrm{~m} / \mathrm{s}$ which is also well within the formal uncertainties derived for each data point. Thus, we conclude that the atmospheric lines are well suited as a reference to trace the RV of the science target. We also note here that the spectral resolution of the emission lines is limited by the slit width to $R \sim 50000$ while the resolution of the object spectrum is more dependent on the true FWHM of the PSF in the slit, thus providing a higher resolution.

Finally we measure the RV of the photospheric lines of MS Vel and in parallel the atmospheric lines in absorption, as outlined above, by cross-correlating the masked spectra of the 


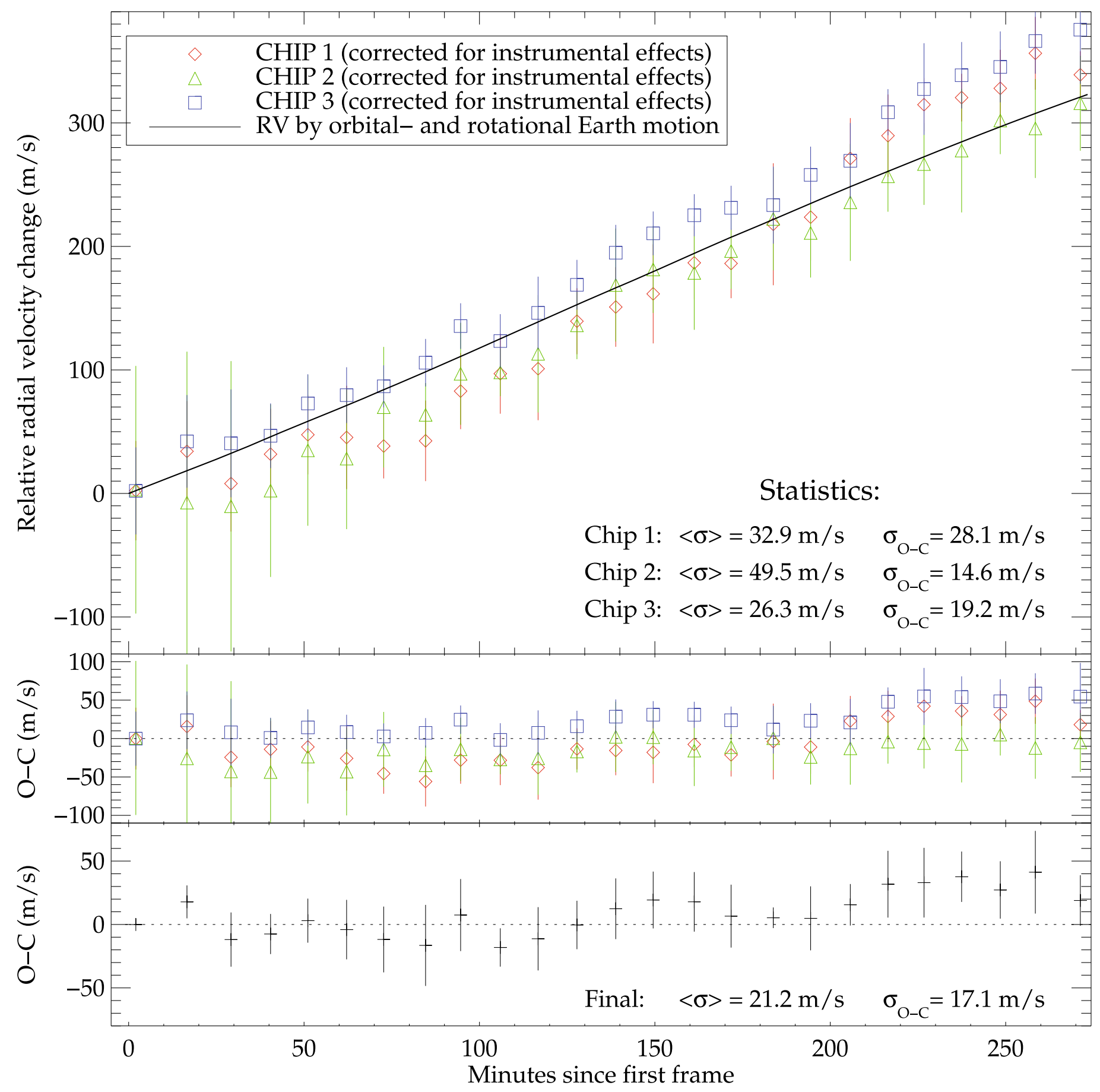

Fig. 6. Radial velocity of the science target - MS Vel - after subtraction of the radial velocity of telluric absorption lines imprinted in the same spectrum (see Fig. 1). The linear trend is modeled by the barycentric and rotational velocity of the Earth during the observation. In the subpanels the data after subtraction of the model is shown in terms of observed - computed $(\mathrm{O}-\mathrm{C})$, first separately for each of the three chips analyzed here, then for the mean of all three chips. The standard deviation of the mean was used to derive formal error bars. See text for details.

source against the first measured spectrum and the unmasked spectra against a template of the atmospheric absorption lines. Both line-systems show a strong near-linear trend with time, peaking at $\sim 1.5 \mathrm{~km} \mathrm{~s}^{-1}$ after $4.5 \mathrm{~h}$. This trend is most likely caused by residuals from the correction of the chromatic refraction (see point 4 in Sect. 1). In addition, the nodding pattern induces sudden jumps of up to $500 \mathrm{~m} / \mathrm{s}$ (1/3 pixel) between the two nodding positions due to the slit curvature and a slight rotation of chips one and two in the focal plane.
Subtracting the RV of the atmospheric absorption lines (that suffer the same displacements) from the RV measured in the masked source spectrum cancels out most of the effects of the nodding displacement and the source misalignment due the refraction correction. A linear trend is left in the opposite direction to the trend observed in the individual spectra of the source, peaking at $300 \mathrm{~m} / \mathrm{s}$ after $4.5 \mathrm{~h}$. The results are displayed in Fig. 6 . Again, we combine the 12 spectra from each of the 25 nodding cycles into one data point. 
The remaining trend can be modeled by the barycentric motion and rotation of the Earth. Subtracting this trend leaves residuals of the order of approx. $\pm 20 \mathrm{~m} / \mathrm{s}$. The residuals appear not noise-like, even though the formal uncertainties of the RV points would account for most of the amplitude of these residuals, but rather show a smooth and semi-periodic behaviour. These residuals are not correlated with the residuals in the atmospheric emission lines (see Fig. 5) making it unlikely that the variability of atmospheric lines, e.g. by wind drifts, are responsible for the residuals in the data. It is also very unlikely that the source itself shows a RV variability within one or two hours, given the large radius of the giant star. Hence, we assign the residuals to a general noise floor limiting the RV precision to this level, most likely being due to the data analysis rather than intrinsic to instrumental instabilities.

We note that our analysis does not take changes in the lineprofile into account. This is an essential part of the analysis when aiming for very high radial velocity precision (Valenti et al. 1995; Butler et al. 1996) using in-situ measurements through a gascell. The method involves a template spectrum of the gascell with much higher resolution than the one used for the actual science measurements. This template spectrum is used to deconvolve the measured lines to determine the instrumental profile. We did not have such a spectrum of our $\mathrm{N}_{2} \mathrm{O}$ gascell or for the telluric lines. Hence, this shortcoming can easily account for the remaining residuals. Moreover, the masking technique to separate the telluric and stellar lines in the individual spectra is not perfect for highly blended line systems, as is the case here.

\section{Conclusions}

We have performed the first radial velocity stability test of CRIRES with $4.5 \mathrm{~h}$ of on-sky measurements of the giant MS Vel at $4100 \mathrm{~nm}$. The dispersive parts of the spectrograph were electronically stabilised, thus decoupling the problems of functional repeatability, spectrograph stability and tracking errors. Besides this non-standard setting we used the spectrograph as offered to the community, which demonstrates that even at this very early stage of its life, CRIRES is a stable instrument, ready to measure radial velocities with high precision including a potential for even higher performance when using an appropriate gas as the local RV zero point.

We could demonstrate that CRIRES shows only a slow drift in its intrinsic radial velocity zero point of $\sim 60 \mathrm{~m} / \mathrm{s}$ in $4.5 \mathrm{~h}$. This drift is fully compensatable using a $\mathrm{N}_{2} \mathrm{O}$ gascell in calibration mode or telluric lines as a local rest frame. Moreover, if our assumption holds that the drift is due to a small change in temperature of the grating (by approx. 1/20 K), it will not appear in any later measurements as the grating is now actively stabilised to approx. $1 \mathrm{mK}$.

The telluric lines in emission observed with a resolution of $R \sim 50000$ are stable in RV to a level of $\leq \pm 10 \mathrm{~m} / \mathrm{s}$ and are therefore suitable as a local rest frame for high precision RV measurements. For the relative radial velocity of our science target, MS Vel, we could recover the $300 \mathrm{~m} / \mathrm{s}$ drift induced by rotational and barycentric Earth motion. The remaining residuals (rms) in the stellar spectra are $\leq \pm 22 \mathrm{~m} / \mathrm{s}$.

We can conclude that the telluric lines imprinted on the spectra of the science target are not a limiting factor for precise $\mathrm{RV}$ measurements in the near infrared but can instead be used as a substitute for a gascell providing the necessary rest frame in cases where no suitable gas can be found or the interesting stellar features are in regions of high telluric line density. The first scientific results using this method are presented in Huelamo et al. (2008).

The influence of mismatches between the diffraction limited FWHM of the PSF and the typical slit width in combination with dynamic (i.e. tracking induced) misalignments of the PSF with respect to the slit centre are substantial. However, these problems can be compensated for when telluric or gascell lines are measured simultaneously with the source and used as a local rest frame. This method is well known and widely used in the optical, where iodine lines are used as the local restframe. Given the high rate of close blends of telluric lines and photospheric lines from our science target and taking the limitations of our analysis into account, we see a clear margin for a gain in performance. Especially, the use of a gascell, well characterised by a high resolution laboratory spectrum $(R \gg 100000)$, should provide a better local rest frame when aiming for a RV precision below $\approx 20 \mathrm{~m} / \mathrm{s}$. The gas has to exhibit a high and stable line density in regions where the science target also shows a rich spectrum. To avoid blends with telluric lines, rare isotope enhanced gases could be used which would elegantly avoid problems with blending telluric features.

In the example presented here - as for all conceivable RV applications of CRIRES - rotational vibrational transitions of molecules in the vibrational ground-state are used. These gases behave very close to ideal gases. This implies that the density of molecules in the beam is invariable and relatively easy controllable. This situation is fundamentally different in the iodine gascells used for radial velocity work. There iodine vapour is used in a heated cell and condensation on cooler parts can result in strong changes of the vapour pressure. Therefore, in our case it is safe to assume that the transition frequencies are very close to those determined by NIST using heterodyne-techniques relative to the caesium time standard. The spectra are relatively simple and lines which may consist of a blend can be excluded. The only conceivable process that may change transition frequencies in the gascell in the case of an ideal gas is pressure shift. The pressure may change due to the external temperature, which will be monitored. It has been estimated that this effect is less than $1 \mathrm{~m} / \mathrm{s}$. A more serious problem is pressure shift by air, if the gascell has a leak. Figure 2b in Glenar et al. (1988) gives an example. $\mathrm{A} \mathrm{PH}_{3}$-gascell line in the presence of a leak is measured relative to a gain-stabilised $\mathrm{CO}_{2}$-laser in a heterodyne setup. The kinks in the $\mathrm{PH}_{3}$ trace correspond to refillings of the cell. To control this problem, the pressure in the CRIRES gascells will be monitored as part of regular operations.

A gascell filled at low pressure with species for which NIST has performed a heterodyne reference measurement relative to the time standard (caesium-clock) in principle provides an absolute secondary frequency standard. To that end CRIRES measurements are linked in an absolute and traceable way to the time standard. This is, strictly speaking, not an actual experimental proof, but does indeed allow for a "cm/s"-type calibration of the spectrograph. Indeed e.g. a spurious blend of gascell lines with weak telluric lines may produce artificial shifts exceeding this value. Experts stress that the frequency calibration of spectrographs breaks down at a precision of $10^{10}$ and only heterodyne techniques can provide this precision (Theodor Haensch, priv. communication).

This paper was aimed at demonstrating the short-term stability of CRIRES and the implications when observing with an underfilled entrance slit. The long-term stability of the instrument, especially important for RV studies, will be considered in a later paper. 
Acknowledgements. We are grateful to all colleagues involved in the development, installation and commissioning of CRIRES. A.S. acknowledges financial support from the Deutsche Forschungsgemeinschaft under DFG RE 1664/4-1.

\section{References}

Adelman, S. J. 2001, VizieR Online Data Catalog, 336, 70297

Aringer, B., Höfner, S., Wiedemann, G., et al. 1999, A\&A, 342, 799

Arsenault, R., Donaldson, R., Dupuy, C., et al. 2004, SPIE, 5490, 47

Butler, R. P., Marcy, G. W., Williams, E., et al. 1996, PASP, 108, 500

Clough, S. A., Kneizys, F. X., Rothman, L. S., \& Gallery, W. O. 1981, Proc. SPIE, 277, 152

Clough, S. A., Iacono, M. J., \& Moncet, J.-L. 1992, J. Geophys. Res., 97, 15761 Deming, D., Espenak, F., Jennings, D. E., Brault, J. W., \& Wagner, J. 1987, ApJ, 316, 771

Egan, M. P., Price, S. D., Kraemer, K. E., et al. 2003, VizieR Online Data Catalog, 5114, 0

Egret, D. 1980, Bulletin d'Information du Centre de Données Stellaires, 18, 82 Freytag, B., \& Höfner, S. 2008, A\&A, 483, 571
Glenar, D. A., Kaufl, H. U., Deming, D., Kostiuk, T., \& Mumma, M. J. 1988, Advances in Helio- and Asteroseismology, 123, 481

Hauschildt, P., \& Baron, E. 2005, Mem. Soc. Astron. Ital. Suppl., 7, 140

Hekker, S., Snellen, I. A. G., Aerts, C., et al. 2008, A\&A, 480, 215

Huelamo, N., et al. 2008, ArXiv e-prints, 808 [arXiv: 0808.2386]

Käufl, H. U., Ballester, P., Biereichel, P., et al. 2004, SPIE, 5492, 1218

Käufl, H. U., Esteves, R., Fedrigo, E., et al. 2006a, The Messenger, 124, 2

Käufl, H. U., Esteves, R., Fedrigo, E., et al. 2006b, The Messenger, 126, 32

Koen, C., \& Laney, D. 2000, MNRAS, 311, 636

Li, C.-H., Benedick, A. J., Fendel, P., et al. 2008, Nature, 452, 610

Makarov, V., Bastian, U., Hoeg, E., Grossmann, V., \& Wicenec, A. 1994, Informational Bulletin on Variable Stars, 4118, 1

Maki, A. G., \& Wells, J. S. 1992, NISTJ, 97, 409 http: //physics .nist .gov/ PhysRefData/wavenum/html/contents.html

Paufique, J., et al. 2006, [arXiv: astro-ph/0608011]

Percy, J. R., Mashintsova, M., Nasui, C. O., et al. 2008, PASP, 120, 523

Pojmanski, G. 2002, Acta Astronomica, 52, 397

Rothman, L. S., Jacquemart, D., Barbe, A., et al. 2005, J. Quant. Spec. Radiat. Transf., 96, 139

Valenti, J. A., Butler, R. P., \& Marcy, G. W. 1995, PASP, 107, 966 